\title{
THE TEMPLATES OF NONSINGULAR SMALE FLOWS ON THREE MANIFOLDS
}

\author{
BIN YU
}

\begin{abstract}
In this paper, we first discuss some connections between template theory and the description of basic sets of Smale flows on 3-manifolds due to F. Béguin and C. Bonatti. The main tools we use are symbolic dynamics, template moves and some combinatorial surgeries. Second, we obtain some relationship between the surgeries and the number of $S^{1} \times S^{2}$ factors of $M$ for a nonsingular Smale flow on a given closed orientable 3-manifold $M$. Besides these, we also prove that any template $T$ can model a basic set $\Lambda$ of a nonsingular Smale flow on $n S^{1} \times S^{2}$ for some positive integer $n$.
\end{abstract}

\section{INTRODUCTION}

The paper is about using template theory to understand nonsingular Smale flows (abbreviated as NS flows) on 3-manifolds.

On the level of topological equivalence, dynamics of general structually stable flow is complicated and not much is know, even in dimension 3. However, if we restrict ourselves to a special class, various methods apply. For example, nonsingular Morse Smale flows (abbreviated as NMS flows) have been effectively studied by associating NMS flows with a kind of combinational tool, i.e., round handle decomposition, see [1], [16] and [24]. Here an NMS flow is a structurally stable flow whose nonwandering set is exactly composed of finite closed orbits.

A larger class than NMS flows is NS flows. NS flows were first introduced by J. Franks in the 1980s, see [7], [8], and [11]. An NS flow is a structurally stable flow with one-dimensional invariant sets and without singularities. For general NS flows, the situation becomes more complicated than NMS flows. A theorem of Bowen [3] says that a one-dimensional hyperbolic basic set $\Lambda$ must be topologically equivalent to a suspension of a subshift of finite type (abbreviated as SSFT). Therefore, symbolic dynamics is very useful in the study of one-dimensional hyperbolic basic set. However, it doesn't provide any embedding information. J. Franks ([7], [8], [11]) used homology and graphs to describe some embedding information of NS flows on 3-manifolds.

To study NS flows on 3-manifolds extensively, a natural idea is to discuss a kind of neighborhoods of basic sets and then study how to glue these neighborhoods together.

Template theory (see Section 2.1) provides a kind of neighborhoods of onedimensional hyperbolic basic sets, i.e., thickened templates. Thickened templates are useful to the study of NS flows on 3-manifolds because:

Date: ????? ?, 2010 and, in revised form, ????? ?, 201 ?.

2000 Mathematics Subject Classification. 57N10, 58K05; 37E99, 37D45.

Key words and phrases. 3-manifold, nonsingular Smale flow, template, filtrating neighborhood.

The author was supported in part by the National NSF of China (grant no. 11001202). 
(1) A thickened template is homeomorphic to a handlebody;

(2) Using a thickened template, one can easily read the symbolic dynamics of a basic set and describe the knot types of the closed orbits in the basic set.

M. Sullivan [23] and the author [26] used thickened templates to discuss a special type of NS flows on 3-manifolds. However, it seems hopeless to establish a general framework for NS flows on 3-manifolds by using thickened templates due to the following two facts:

(1) It is difficult to describe the entrance set and the exit set in the boundary of a thickened template;

(2) Many different templates can be used to model a given nontrivial basic set.

In [2], F. Béguin and C. Bonatti gave several concepts and results to describe the behavior of a given nontrivial basic set. An introduction to their work can be found in Section 2.3. Their work provided another kind of neighborhoods of nontrivial basic sets on 3-manifolds, i.e., filtrating neighborhoods. We note that the same concept was also discovered by J. Franks in [11], who named it "building block". Filtrating neighborhoods are useful because:

(1) The filtrating neighborhood of $\Lambda$ is unique up to topological equivalence for a given nontrivial basic set $\Lambda$;

(2) Filtrating neighborhoods are natural chunks for the reconstruction of the underlying 3-manifold with a Smale flow. See [11] and [20].

In spite of these advantages, it is difficult to study the topological structures of filtrating neighborhoods systematically.

In this paper, we first give a structure theorem (see Theorem 4.4) to show some connections between the filtrating neighborhood and a thickened template for a given nontrivial basic set. The structure is described by some parameters $\left(g_{i}, k_{i}, t\right)$. In this process, we also discuss some connections between template theory and some concepts proposed by F. Béguin and C. Bonatti (see Theorem 3.3 and Theorem 4.3). Our main tools are some combinatorial surgeries, i.e., surgeries on edge graphs, template moves and attaching thickened surfaces with flows.

In Theorem 4.5, we find that for an NS flow on a 3-manifold $M$, the parameters $\left(g_{i}, k_{i}\right)$ are bounded by a topological invariant of 3-manifolds, i.e., the number of the $S^{1} \times S^{2}$ factors of $M$ (see Theorem 4.5). As a corollary (Corollary 4.6), given a template $T$ and a closed orientable 3-manifold $M$, there exist at most finitely many filtrating neighborhoods (up to topological equivalence) modeled by $T$ in an NS flow on $M$. As an application, we give an example (Example 4.7) to discuss the realization of a special type of NS flows on 3-manifolds. Example 4.7 also shows that although the topological type of filtrating neighborhood (given $T$ and $M$ ) is finite, there are infinitely many possible different ways to embed some filtrating neighborhood of $T$ as a filtrating neighborhood of an NS flow on $M$.

Another interesting problem is the realization of one-dimensional basic sets in NS flows on 3-manifolds, which will be the topic in the last section. If the basic sets are described by SSFT, the realization problem is considered in [19] and [9]. The realization problem of SSFT is completely solved in the sense that for any SSFT and any closed orientable 3-manifold $M$, there is an NS flow $\phi_{t}$ on $M$ such that the SSFT can be realized as a basic set of $\phi_{t}$ (see [11] and Proposition 6.1 in [7]). If the basic sets are described by a template, Meleshuk [17] proved that there exists some 3-manifold admitting an NS flow such that there exists a basic set of the NS 
flow modeled by the template. It is natural to ask: for a given template $T$, which 3 -manifolds admit (or do not admit) an NS flow with a basic set modeled by $T$ ? The author [27], proved that:

(1) If a closed orientable 3-manifold $M$ admits an NS flow with a basic set modeled by $T$, then $M=M^{\prime} \sharp g(T) S^{1} \times S^{2}$. Here $g(T)$ is the genus of $T$, see $[27]$.

(2) There exists a closed orientable 3-manifold $M^{\prime}$ such that $M=M^{\prime} \sharp g(T) S^{1} \times$ $S^{2}$ admits an NS flow with a basic set modeled by $T$.

In the last section, we prove that any template $T$ can model a basic set $\Lambda$ of an NS flow $\psi_{t}$ on $n S^{1} \times S^{2}$ for some positive integer $n$ (obviously $n \geq g(T)$ ), see Theorem 5.5 .

\section{Preliminaries}

Basic definitions and facts about dynamical systems can be found in [21].

2.1. Template. A Template $(T, \phi)$ is a smooth branched 2-manifold $T$, constructed from two types of charts, called joining charts and splitting charts, together with a semi-flow. A semi-flow is the same as flow except that one cannot back up uniquely. The semi-flows are indicated by arrows on charts in Figure 1. The gluing maps between charts must respect the semi-flow and act linearly on the edges.

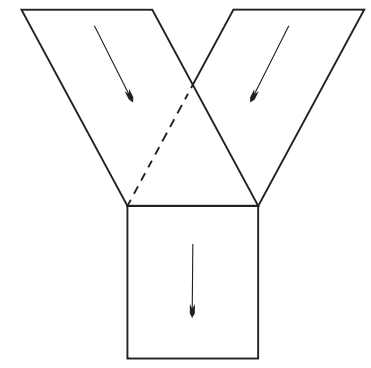

(a) joining chart

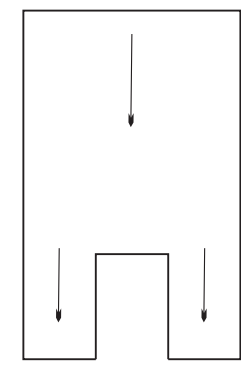

(b) splitting chart

FIGURE 1.

The following template theorem of R. Williams and J. Birman ([5], [6], [13]) shows the importance of template theory to the study of flows on 3-manifolds.

Theorem 2.1 (the template theorem). Let $\phi_{t}$ be a smooth flow on a 3-manifold $M$ with a hyperbolic basic set. The link of closed orbits $L_{\phi}$ is in bijective correspondence with the link of closed orbits $L_{T}$ on a particular embedded template $T \subset M$ (with $L_{T}$ containing at most two extraneous orbits).

Remark 2.2. In Theorem 2.1, if the topological dimension of the basic set is 1, then the correspondence is exactly bijective without any extraneous orbits.

Now we introduce template moves, which were introduced by M. Sullivan and the others ([13], [14]). 
Definition 2.3 (template moves). For a template, as Figure 2 shows, the move in Figure 2 (a) is called a slide move; the move in Figure 2 (b) is called a split move. Slide moves, split moves and their converse moves are collectively referred to as template moves.

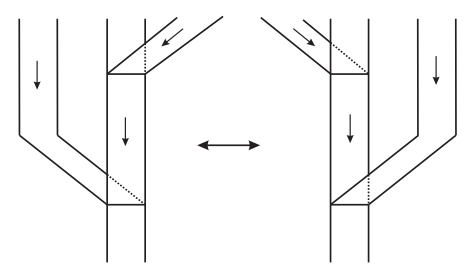

(a) slide move

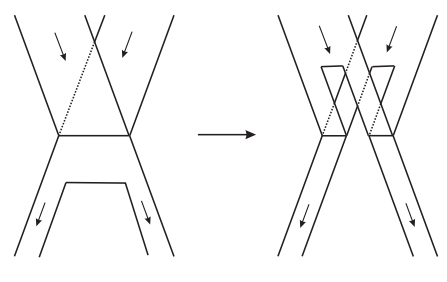

(b) split move

FIGURE 2.

We now briefly recall the construction of the templates of dimension 1 hyperbolic basic sets, which is useful to our discussion. More details can be found in [13].

Let $B$ be a dimension 1 hyperbolic basic set of a flow $\phi_{t}$. By Theorem 2.9, $B$ is conjugate to an SSFT. Furthermore, Bowen and Walters [4] showed that there exists a finite union of disjoint discs such that the Poincaré return map on these discs satisfies the condition of Markov partitions. Throughout this paper, this union of disjoint discs is called a cross-section. The neighborhood of one of the above discs is known as a Markov flowbox neighborhood. The incoming and outgoing flowboxes near a flowbox are shown in Figure 3(a). A flowbox neighborhood is used to model the flow on the neighborhood of one of the above discs. With this in mind, we can normalize it to Figure 3(b) through a small perturbation.

Further, we decompose the perturbed Markov flowbox into some joining flowboxes and some splitting flowboxes in Figure 4. The gluing maps between simple flowboxes must respect the flow and act linearly on the boundaries.

For a given Markov flowbox neighborhood of $B$ constructed above, we can "crush" the stable foliations to $T$ which is a branched manifold with semiflow. Locally, we obtain joining (resp. splitting) charts from joining (resp. splitting) flowboxes and gluing maps between charts from gluing maps between simple flowboxes. It follows naturally from the definition that the gluing maps between charts respect the flow and act linearly on the edges.

Therefore, we obtain an embedded template which models the dimension 1 basic set.

Remark 2.4. A template move changes the topological type of the template but that it is done in such a way as to not change the basic set. 


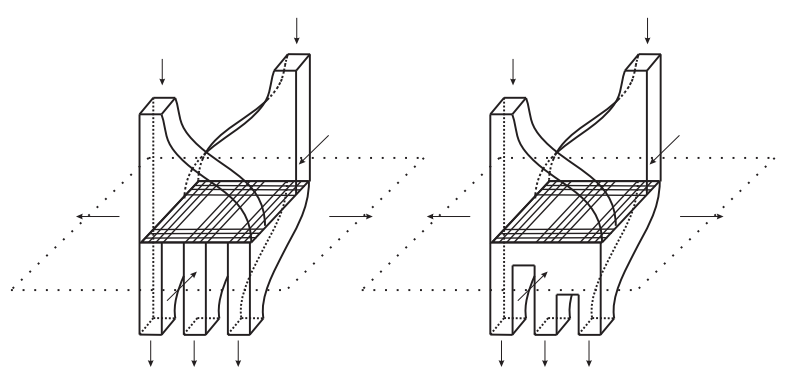

(a)

(b)

FiguRE 3 .

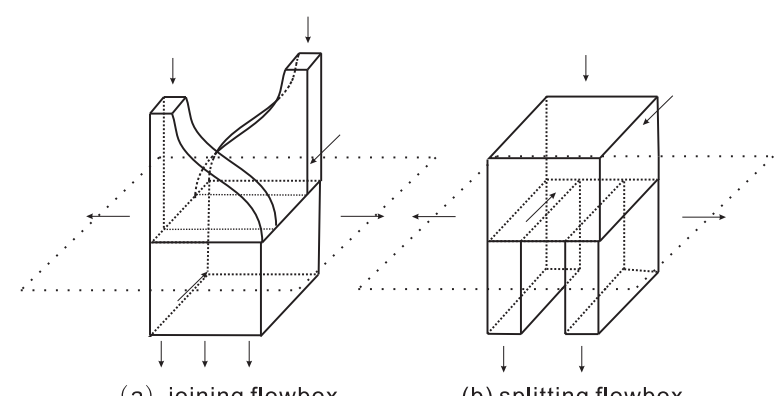

(a) joining flowbox

FIGURE 4.

2.2. Symbolic dynamics and State splitting of edge graphs. All the definitions and the facts in this subsection can be found in [8], [15].

Let $\Sigma_{n}=\left\{s=\left(\ldots, s_{-1}, s_{0}, s_{1}, \ldots\right) \mid s_{j} \in\{1, \ldots, n\}, \forall j \in \mathbb{Z}\right\}$. We can define a distance on $\Sigma_{n}$ as follows: for any $s, t \in \Sigma_{n}, d(s, t)=\Sigma_{j=-\infty}^{+\infty} \frac{\delta\left(s_{j}, t_{j}\right)}{4|j|}$ where $\delta(a, b)=\left\{\begin{array}{lll}0, & \text { if } & a=b \\ 1, & \text { if } & a \neq b\end{array} . \quad \Sigma_{n}\right.$ is called a two sided shift space. A homeomorphism $\sigma$ on $\Sigma_{n}$ is defined as following. For any $s \in \Sigma_{n}$, let $t=\sigma(s)$ where $t_{j}=s_{j+1} \cdot\left(\Sigma_{n}, \sigma\right)$ is called a full two sided shift.

Let $A=\left(a_{i j}\right)_{n \times n}, a_{i j} \in\{0,1\}$. $A$ is called a transition matrix. $\Sigma_{A}$ is defined by $\Sigma_{A}=\left\{s=\left(s_{j}\right) \in \Sigma_{n} \mid a_{s_{j}, s_{j+1}}=1, \forall j \in \mathbb{Z}\right\}$. We denote $\left.\sigma\right|_{\Sigma_{A}}$ by $\sigma_{A} \cdot\left(\Sigma_{A}, \sigma_{A}\right)$ is called a subshift of finite type.

Actually, we can define a subshift of finite type for any matrix $A=\left(a_{i j}\right)_{n \times n}, a_{i j} \in$ $\mathbb{Z}^{+}$(such a matrix is called an adjacent matrix). For this purpose, we need to introduce two concepts, vertex graphs and edge graphs. For an adjacent matrix $A$, we take $n$ vertexes $v_{1}, v_{2}, \ldots, v_{n}$, then attach $a_{i j}$ oriented edges from $v_{i}$ to $v_{j}$ for any $i, j \in\{1, \ldots, n\}$. Therefore, we get an oriented graph $G$ which is called the vertex graph of $A$. Denote the edges of the vertex graph $G$ by $e_{1}, e_{2}, \ldots, e_{m}$. For the edge graph $F$ of $A$, take the edges of $G$ as the vertexes, which are still denoted by $e_{1}, e_{2}, \ldots, e_{m}$ and join $e_{i}$ to $e_{j}$ if there exists a vertex $p$ in $G$ such that $e_{i}$ starts from $p$ and $e_{j}$ terminates at $p$. Obviously, for two given vertexes $e_{i}, e_{j}$ in $F$, there exists at most one edge which starts from $e_{i}$ and terminates at $e_{j} . F$ determines a unique transition matrix $A^{\prime}$. $A^{\prime}$ determines a subshift of finite type, which is called the edge shift of $A$. 
For two adjacent matrixes $A$ and $B$, let $X_{A}$ and $X_{B}$ be the edge shifts of $A$ and $B$ respectively. It is natural to ask when $X_{A}$ is conjugate to $X_{B}$. In [25], R. Williams gave a necessary and sufficient condition by using adjacent matrix. We state this condition by using edge graphs, see [15].

Definition 2.5. (1) Let $G$ be an edge graph with vertex set $V$ and edge set $\varepsilon$. For a vertex $v \in V$, we divide $v$ into $v_{1}$ and $v_{2}$, split outgoing edges of $v$ and copy incoming edges of $v$ to obtain a new graph. This surgery is called an out-split, see Figure 5. Reversing the orientation of $G$, we obtain a new graph $G_{1}$. Doing an out-split surgery on $G_{1}$, we obtain a new graph $G_{2}$. Then reversing the orientation of $G_{2}$, we obtain a new graph $G^{\prime}$. We call the surgery from $G$ to $G^{\prime}$ an in-split.

(2) The converse surgery of an out-split (resp. in-split) is called an out-amalgamation (resp. in-amalgamation).

(3) By dividing an oriented edge of an edge graph $G$ to two oriented (the same orientation) edges by increasing a vertex to the oriented edge of $G$, we obtain a new graph $G^{\prime}$. This surgery is called an expanding surgery.

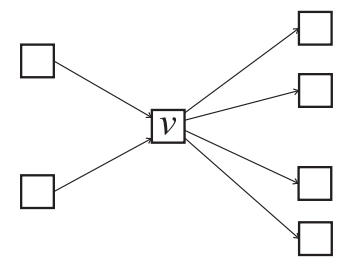

(a)

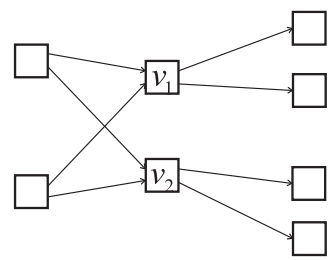

(b)

Figure 5 .

Theorem 2.6. Every conjugacy from one edge shift to another is a composition of finite steps of splitting surgeries and amalgamation surgeries.

For SSFT, Parry and D. Sullivan [18] gave a complete classification up to topological equivalence by using adjacent matrix. Moreover, J. Franks [10] found a complete set of computable algebraic invariants. Here we only state this kind of result by using edge graph, see [15].

Theorem 2.7. Two SSFT given by edge graphs $G$ and $F$ are topologically equivalent if and only if there exists a finite sequence of edge graphs $G=G_{0}, G_{1}, \ldots, G_{r}=F$ satisfying $G_{i} \sim_{s} G_{i+1}$ or $G_{i} \sim_{e} G_{i+1}$ for $i=0, \ldots, r-1$.

Here $G_{i} \sim_{s} G_{i+1}$ means that $G_{i+1}$ can be obtained from $G_{i}$ by a splitting or an amalgamation. $G_{i} \sim_{e} G_{i+1}$ means that $G_{i+1}$ can be obtained from $G_{i}$ by an expanding surgery or the converse of an expanding surgery.

\subsection{NS flows on three manifolds.}

Definition 2.8. A smooth flow $\phi_{t}$ on a compact manifold $M$ is called a Smale flow if:

(1) the chain recurrent set $R\left(\phi_{t}\right)$ has hyperbolic structure;

(2) $\operatorname{dim}\left(R\left(\phi_{t}\right)\right) \leq 1$; 
(3) $\phi_{t}$ satisfies the transverse condition.

If a Smale flow $\phi_{t}$ has no singularity, we call $\phi_{t}$ a nonsingular Smale flow (an $N S$ flow). In particular, if $R\left(\phi_{t}\right)$ consists entirely of closed orbits, $\phi_{t}$ is called a nonsingular Morse Smale flow (an NMS flow).

The following theorem is due to Smale [22] and Bowen [3].

Theorem 2.9. The chain recurrent set $R\left(\phi_{t}\right)$ of an $N S$ flow $\phi_{t}$ on a compact manifold $M$ satisfies:

(1) (Spectral decomposition) $R\left(\phi_{t}\right)=\Lambda_{1} \sqcup \ldots \sqcup \Lambda_{n}$. Here each $\Lambda_{i}(i=1, . ., n)$ is called a basic set, i.e., $\Lambda_{i}$ is an invariant subset of $R\left(\phi_{t}\right)$ and is a closure of an orbit;

(2) $\Lambda_{i}$ is a closed orbit or $\Lambda_{i}$ satisfies that $\phi_{t}$ restricted to $\Lambda_{i}$ is topologically equivalent to an SSFT.

Now we introduce the descriptions of basic sets of Smale flows on 3-manifolds by F. Beguin and C. Bonatti [2].

Let $\phi_{t}$ and $\varphi_{t}$ be two smooth flows with compact invariant sets $K$ and $L$ respectively. $\left(\phi_{t}, K\right)$ and $\left(\varphi_{t}, L\right)$ are said to be equivalent if and only if there exists a neighborhood $U$ of $K$ and a neighborhood $V$ of $L$ such that the restrictions of $\phi_{t}$ to $U$ and $\varphi_{t}$ to $V$ are topologically equivalent via a homeomorphism sending $K$ to $L$. The germ $\left[\phi_{t}, K\right]$ is the equivalence class represented by $\left(\phi_{t}, K\right)$.

Let $\phi_{t}$ be a smooth flow on a closed orientable 3-manifold $M$ and let $K$ be a saddle set of $\phi_{t}$. By a model of the germ $\left[\phi_{t}, K\right]$, we mean a pair $\left(\varphi_{t}, N\right)$, where $N$ is a compact orientable 3 -manifold and $\varphi_{t}$ is a smooth flow on $N$ transverse to the boundary, such that:

(1) The maximal invariant set for $\varphi_{t}$ in $N$ is a saddle set $K_{\varphi_{t}}$ such that the germ $\left[\varphi_{t}, K_{\varphi_{t}}\right]$ is equal to the germ $\left[\phi_{t}, K\right]$.

(2) Denote by $\partial_{1} N$ the union of the connected components of the boundary of $N$ where $\varphi_{t}$ is coming into $N$; then any circle embedded in $\partial_{1} N$ and disjoint with $W^{s}\left(K_{\varphi_{t}}\right)$ bounds a disc in $\partial_{1} N$ which is also disjoint with $W^{s}\left(K_{\varphi_{t}}\right)$.

(3) Any connected component of $N$ contains at least one point of $K_{\varphi_{t}}$.

Theorem 2.10. Given a saddle basic set $K$ of an NS flow $\phi_{t}$ on a closed orientable 3-manifold $M$, there exists a unique (up to topological equivalence) model $\left(\varphi_{t}, N\right)$ of the germ $\left[\phi_{t}, K\right]$.

Theorem 2.11. Let $\phi_{t}$ and $\varphi_{t}$ be two Smale flows on two orientable 3-manifolds $M$ and $N$. Let $K$ and $L$ be two saddle basic sets of $\phi_{t}$ and $\varphi_{t}$ respectively. If the models of $\left[\phi_{t}, K\right]$ and $\left[\varphi_{t}, L\right]$ are topologically equivalent, then there exist invariant neighborhoods $U$ of $K$ and $V$ of $L$ such that the restriction of $\phi_{t}$ to $U$ is topologically equivalent to the restriction of $\varphi_{t}$ to $V$.

A filtrating neighborhood of $K$ is a neighborhood $U$ such that:

(1) $K$ is the maximal invariant set (for $\phi_{t}$ ) in $U$.

(2) The intersection of any orbit of $\phi_{t}$ with $U$ is connected (in other terms, any orbit getting out of $U$ never comes back).

Let $\left(\varphi_{t}, N\right)$ be a model of the germ $\left[\phi_{t}, K\right]$. Let $D_{1}$ and $D_{2}$ be two closed disjoint discs contained in the entrance boundary of $N$ such that the orbits of $D_{1}$ and $D_{2}$ leave $N$ in a finite amount of time. The orbits of these two discs form the union of two cylinders $D^{2} \times[0,1]$ endowed with the vector-field $\frac{\partial}{\partial t}$. Let us cut out of $N$ these 
two cylinders and then paste the two resulting tangent boundary components. By doing so, we have added to $N$ a handle, getting a new 3-manifold still endowed with a vector-field. This surgery is called handle attachment.

Proposition 2.12. If $U$ is a filtrating neighborhood of $K$ such that all connected components of $U$ meet $K$, one can obtain a pair topologically equivalent to $(U, K)$ by modifying $\left(\varphi_{t}, N\right)$ by a finite number of handle attachments of the above type.

\section{Template And Germ}

If we extend a template $T$ in the direction perpendicular to its surface, we obtain a thickened template $\bar{T}$. The semi-flow on $T$ extends to a flow on $\bar{T}$, see Figure 6 . $\partial \bar{T}$ is composed of the entrance set $X$, the exit set $Y$ and the dividing curves set $C$. More details about thickened templates can be found in [17]. Denote $\varphi_{t}^{T}$ by the flow on $\bar{T}$ and $K_{T}$ by the invariant set of $\varphi_{t}^{T}$.

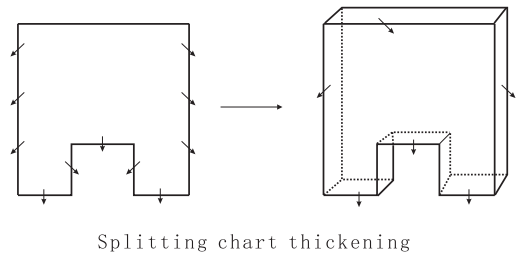

FiguRE 6.

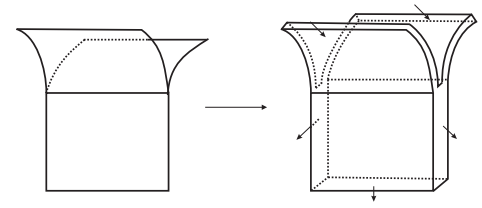

Joining chart thickening

Then, we can define a germ $\left[\varphi_{t}^{T}, K_{T}\right]$ for a template $T$. On the other hand, by Theorem 2.1 (the template theorem), any germ can be represented by a template.

By the proof of Theorem 2.5 in [18], we have the following lemma.

Lemma 3.1. Up to isotopic equivalence, different cross-sections can be exchanged by a finite sequence of unstable direction divisions (or their converses), stable direction divisions (or their converses) and adding parallel cross sections (or their converses).

Now we state the key lemma in this section.

Lemma 3.2. Let $\phi_{t}$ be a smooth flow on a 3-manifold $M$ having a hyperbolic chainrecurrent set which contains a dimension 1 basic set $\Lambda$. Every two templates induced by $\Lambda$ can be exchanged by a finite sequence of template moves.

Proof. Let $T_{1}$ and $T_{2}$ be two templates embedded in $M$ such that both of them model $\Lambda$. Obviously, there exist two cross-sections of $\Lambda$ denoted by $\mathcal{C}_{1}$ and $\mathcal{C}_{2}$ corresponding to $T_{1}$ and $T_{2}$ respectively. If we crush the unstable foliation of $T_{1}$ and $T_{2}$, we can obtain two oriented embedded graphs $G_{1}$ and $G_{2}$. It is easy to see that the two SSFT of the edge shifts defined by both $G_{1}$ and $G_{2}$ are topologically equivalent to $\left.\phi_{t}\right|_{\Lambda}$. Hence $G_{1}$ and $G_{2}$ can be regarded as two edge graphs of $\left.\phi_{t}\right|_{\Lambda}$. Given the relation between $G_{i}$ and $T_{i}$, we call $G_{i}$ the edge graph of $T_{i}$ for $i=1,2$. $G_{1}$ and $G_{2}$ are the so called template edge graphs in the sense that they can be obtained by crushing the unstable foliations of two templates.

By Lemma 3.1 and some observations, we can obtain $\mathcal{C}_{2}$ from $\mathcal{C}_{1}$ by finitely many steps of unstable direction divisions (or their converses), stable direction divisions (or their converses) and adding parallel cross sections (or their converses) such that, 


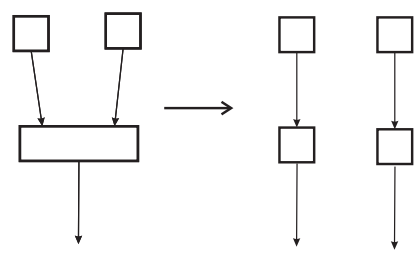

(a) simple in-split

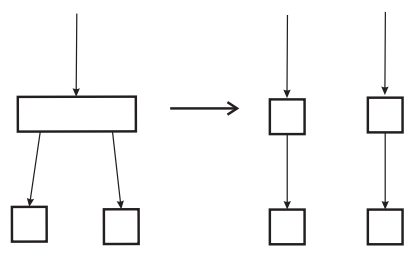

(b) simple out-split

Figure 7.
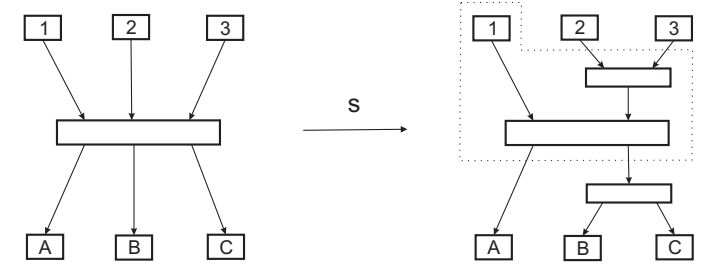

g

$\mathrm{h}$
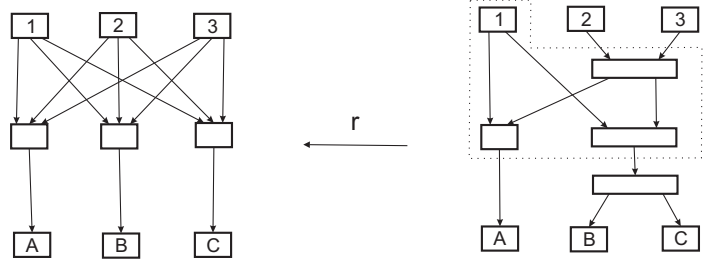

FiguRE 8.

after each step, we obtain a new cross-section of $\Lambda$. Each cross-section corresponds to a Markov flowbox neighborhood (See Section 2.1 or [13]). By crushing the stable and unstable foliations of a Markov flowbox neighborhood, we obtain an edge graph.

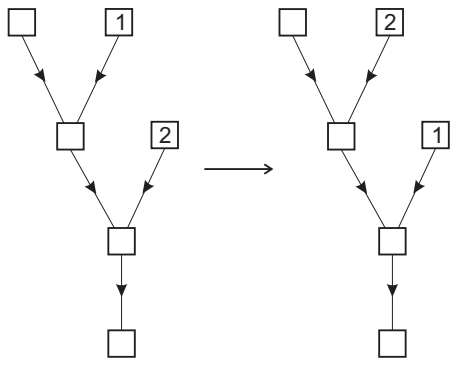

(1)

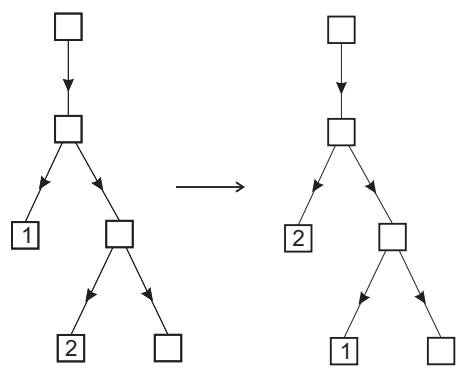

(2)

FIGURE 9. 


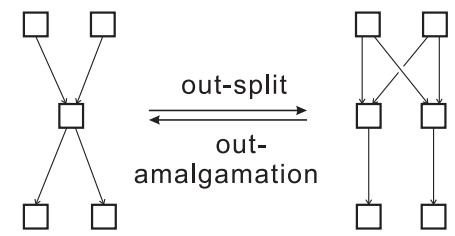

(a)

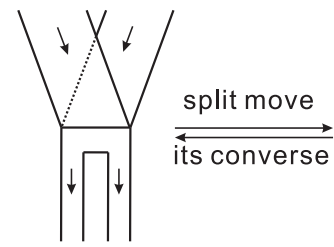

(a') (b)

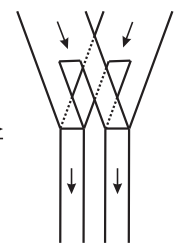

(b')

(1)

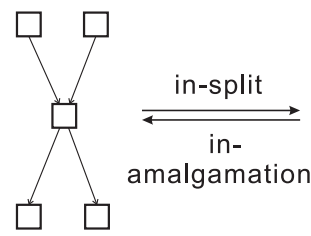

(a)

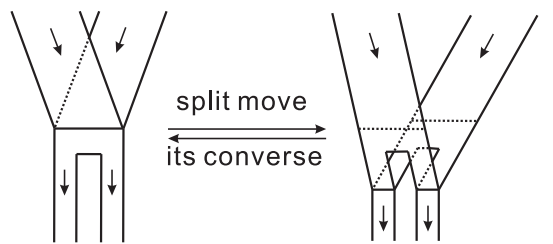

(a')

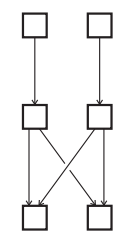

(b)

FiguRE 10.

Obviously, unstable direction division, stable direction division and adding a parallel cross section on cross sections correspond to in-split, out-split and expanding surgery on edge graphs respectively. So, we can obtain $G_{1}$ from $G_{2}$ by $f_{n+1} \circ g_{n} \circ f_{n} \circ \ldots \circ g_{1} \circ f_{1}(n \in \mathbb{N})$. Here each $f_{i}(i=1, \ldots, n+1)$ is a composition of finitely many steps of expanding surgery and/or its converse. and each $g_{i}$ $(i=1, \ldots, n+1)$ is an out-split, an in-split or the inverse of an out-split or in-split. For simplicity, we denote this relation by $G_{1}=f_{n+1} \circ g_{n} \circ f_{n} \circ \ldots \circ g_{1} \circ f_{1}\left(G_{2}\right)$.

Each $g_{i}$ can be written as the composition $r_{i} \circ h_{i} \circ s_{i}$ satisfying the following three conditions:

(1) Both $s_{i} \circ f_{i} \circ \ldots \circ g_{1} \circ f_{1}\left(G_{2}\right)$ and $h_{i} \circ s_{i} \circ f_{i} \circ \ldots \circ g_{1} \circ f_{1}\left(G_{2}\right)$ are template edge graphs.

(2) Both $r_{i}$ and $s_{i}$ are composed of finitely many steps of simple splits (See Figure 7), expanding surgeries and/or their converses.

(3) $h_{i}$ is an out-split, an in-split or the inverse of an out-split or in-split between two template edge graphs.

Figure 8 shows a decomposition of an out-split. In a similar way as Figure 8 shows, each $g_{i}$ admits such a decomposition.

Set $t_{1}=s_{1} \circ f_{1}, t_{i}=s_{i} \circ f_{i} \circ r_{i-1}(i=2, \ldots, n)$ and $t_{i+1}=f_{n+1} \circ r_{n}$. Then $G_{1}=t_{n+1} \circ h_{n} \circ t_{n} \circ \ldots \circ h_{1} \circ t_{1}\left(G_{2}\right)$. Here each $t_{i}(i=1, \ldots, n+1)$ is a composition of finitely many steps of simple splits, expanding surgeries and/or their converses between two template edge graphs. It is easy to show that each $t_{i}$ is a composition of the surgeries in Figure 9, expanding surgeries and the converses of expanding surgeries. The local structure of each $h_{i}$ is shown in Figure 10 (see also Figure 8).

Obviously $t_{n+1} \circ h_{n} \circ t_{n} \circ \ldots \circ h_{1} \circ t_{1}: G_{2} \rightarrow G_{1}$ provides the corresponding transformation from $T_{2}$ to $T_{1}$, i.e., $\mathcal{T}_{n+1} \circ \mathcal{H}_{n} \circ \mathcal{T}_{n} \circ \ldots \circ \mathcal{H}_{1} \circ \mathcal{T}_{1}: T_{2} \rightarrow T_{1}$. Here $\mathcal{T}_{i}$ and $\mathcal{H}_{j}$ correspond to $t_{i}$ and $h_{j}$ respectively. By the constructions of $t_{i}$ and $h_{i}$, each $\mathcal{T}_{i}$ corresponds to a composition of finitely many steps of slide moves and each $\mathcal{H}_{i}$ corresponds to a split move or the converse of a split move (see Figure 10). Therefore, $T_{1}$ and $T_{2}$ can be exchanged by a finite sequence of template moves. 
Theorem 3.3. Let $T_{1}$ and $T_{2}$ be two templates and $\left[\varphi_{t}^{T_{1}}, K_{T_{1}}\right]$ and $\left[\varphi_{t}^{T_{2}}, K_{T_{2}}\right]$ be the germs determined by $T_{1}$ and $T_{2}$ respectively. Then $\left[\varphi_{t}^{T_{1}}, K_{T_{1}}\right]=\left[\varphi_{t}^{T_{2}}, K_{T_{2}}\right]$ if and only if $T_{1}$ and $T_{2}$ can be exchanged by applying a finite sequence of template moves.

Proof. The sufficiency. It suffices to consider only one template move and there are the following two possible cases:

(1) $T_{1}$ is transformed into $T_{2}$ by a slide move;

(2) $T_{1}$ is transformed into $T_{2}$ by a split move.

By the construction of a thickened template, it is easy to show that $\left(T_{1}, \varphi_{t}^{T_{1}}\right)$ is topologically equivalent to $\left(T_{2}, \varphi_{t}^{T_{2}}\right)$ in case (1). Therefore, in this case, $\left[\varphi_{t}^{T_{1}}, K_{T_{1}}\right]=$ $\left[\varphi_{t}^{T_{2}}, K_{T_{2}}\right]$.

In case (2), there exists a template $T_{1}^{\prime} \subset T_{1}$ such that $T_{1}^{\prime}=T_{2}$. So $\left(\overline{T_{1}^{\prime}}, \varphi_{t}^{T_{1}^{\prime}}\right)$ is topologically equivalent to $\left(\overline{T_{2}}, \varphi_{t}^{T_{2}}\right)$ and $K_{T_{1}} \subset \overline{T_{1}^{\prime}} \subset \overline{T_{1}}$. Therefore, $\left[\varphi_{t}^{T_{1}}, K_{T_{1}}\right]=$ $\left[\varphi_{t}^{T_{2}}, K_{T_{2}}\right]$.

The necessity. If $\left[\varphi_{t}^{T_{1}}, K_{T_{1}}\right]=\left[\varphi_{t}^{T_{2}}, K_{T_{2}}\right]$, by the definition of germ, there exists $U_{1}$ and $U_{2}$ which are the neighborhoods of $K_{T_{1}}$ and $K_{T_{2}}$ respectively such that:

(1) $K_{T_{i}} \subset U_{i} \subset \overline{T_{i}}$ for $i=1,2$;

(2) $\left(U_{1},\left.\varphi_{t}^{T_{1}}\right|_{U_{1}}\right)$ is topologically equivalent to $\left(U_{2},\left.\varphi_{t}^{T_{2}}\right|_{U_{2}}\right)$.

Therefore, we can construct templates $T_{1}^{1} \subset U_{1}$ for $\left(U_{1},\left.\varphi_{t}^{T_{1}}\right|_{U_{1}}\right)$ and $T_{2}^{2} \subset U_{2}$ for $\left(U_{2},\left.\varphi_{t}^{T_{2}}\right|_{U_{2}}\right)$ such that $T_{1}^{1}=T_{2}^{2}$. On the other hand, by Lemma 3.2, $T_{i}$ and $T_{i}^{i}(i=1,2)$ can be exchanged by applying a finite sequence of template moves. Therefore, $T_{1}$ and $T_{2}$ can be exchanged by applying a finite sequence of template moves.

\section{Template, Model of Germ and Filtrating Neighborhood}

One may attach a 2-handle along a dividing curve $c$ of a thickened template $\bar{T}$ as shown in Figure 11. Here a 2-handle is a cylinder $D^{2} \times[0,1]$ endowed with the vector field $\frac{\partial}{\partial t}$. The first figure in Figure 11 is a cross-section of $\bar{T}$ along $c$.

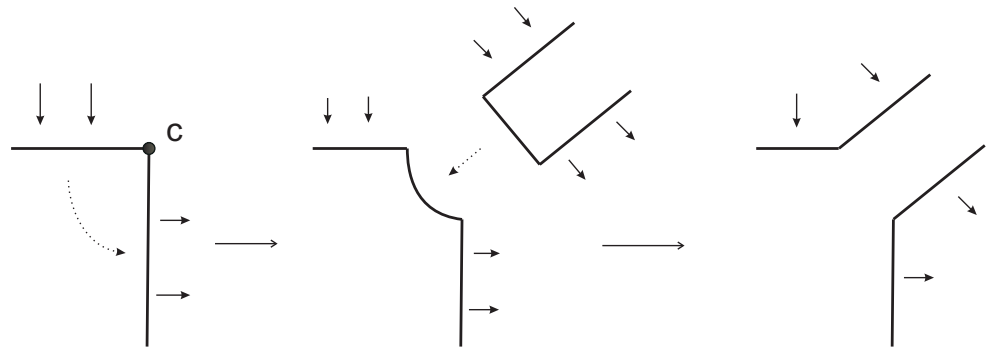

Cross sectional view of attaching a 2-handle to a thickened template along the curve $\mathrm{c}$

Figure 11.

If we repeat the above process for each dividing curve of $\bar{T}$, we obtain a compact 3 -manifold $\bar{N}$ with the flow $\overline{\varphi_{t}}$. Here $\overline{\varphi_{t}}$ is transverse to $\partial \bar{N}$. Obviously, $K_{T} \subset \bar{T} \subset$ $\bar{N}$ is the maximal invariant set for $\overline{\varphi_{t}}$ in $\bar{N}$.

In [12], G. Frank described $W^{s}\left(\varphi_{t}\right) \cap \partial \bar{T}$. We restate his result in the following: 
Theorem 4.1. $W^{s}\left(\varphi_{t}\right) \cap \partial \bar{T}=W^{s}\left(\varphi_{t}\right) \cap X$ where $X$ is the entrance set of $\partial \bar{T}$. It is a lamination $\mathcal{F}$ on $X$ which locally is homeomorphic to $\mathcal{C} \times \mathbb{R}$. Here $\mathcal{C}$ is a Cantor set. In $\mathcal{F}$, there are only a finite number of circles. For any $x \in \mathcal{F}, x \times t$ approaches a circle asymptotically as $t \rightarrow+\infty$ (or $t \rightarrow-\infty$ ). $X-\mathcal{F}$ has infinite connected components. Each connected component of $X-\mathcal{F}$ is homeomorphic to the interior of $D^{2}$.

Remark 4.2. The statement "Each connected ...D" in Theorem 4.1 is not included in the original theorem of G. Frank. But it is easy to prove this fact by the arguments in his paper [12].

Theorem 4.3. Let $\bar{T}$ be a thickened template and let $\bar{N}$ be obtained by attaching 2-handles to each dividing curve. Then $\left(\overline{\varphi_{t}}, \bar{N}\right)$ is the model of the germ $\left[\varphi_{t}^{T}, K_{T}\right]$.

Proof. $\left(\overline{\varphi_{t}}, \bar{N}\right)$ obviously satisfies (1) and (3) in the definition of the model of a germ. Therefore, it is sufficient to prove that $\left(\overline{\varphi_{t}}, \bar{N}\right)$ satisfies $(2)$ of the definition.

For any circle $c$ in $\partial_{1} \bar{N}$ which is disjoint from $W^{s}\left(\overline{\varphi_{t}}\right)$, at least one of the following three situations occurs. Here $\partial_{1} \bar{N}$ is the entrance set of $\left(\overline{\varphi_{t}}, \bar{N}\right)$.

Case 1: $c \subset D^{2} \times 0 \sqcup D^{2} \times 1$ for some attached 2-handle $D^{2} \times[0,1]$. Then obviously $c$ bounds a disk in $\left(D^{2} \times 0 \sqcup D^{2} \times 1\right) \subset \partial_{1} \bar{N}$. It is easy to see that the disk is disjoint from $W^{s}\left(\overline{\varphi_{t}}\right)$.

Case 2: $c \subset X \subset \partial_{1} \bar{N}$ and $c \cap W^{s}\left(\overline{\varphi_{t}}\right)=\emptyset$. Obviously $c \cap W^{s}\left(\varphi_{t}^{T}\right)=\emptyset$. By Theorem 4.1, $c$ is in the interior of a disk which belongs to $X$ and is disjoint from $W^{s}\left(\overline{\varphi_{t}}\right)$. Therefore, $c$ bounds a disk in $X \subset \partial_{1} \bar{N}$. Moreover, the disk is disjoint from $W^{s}\left(\overline{\varphi_{t}}\right)$.

Case 3: $c \cap X \neq \emptyset$ and $c \cap\{2-$ handles $\} \neq \emptyset$. In this case, there exists an isotopy map $F_{t}(x): S^{1} \rightarrow \partial_{1} \bar{N}(t \in[0,1])$ such that $F_{0}\left(S^{1}\right)=c, F_{1}\left(S^{1}\right)=c_{1}$ and $F_{[0,1]}\left(S^{1}\right) \cap W^{s}\left(\overline{\varphi_{t}}\right)=\emptyset$, where $c_{1}$ is a simple closed curve in $X$.

As in Case 2, $c_{1}$ bounds a disk $D_{1} \subset \partial \bar{N}$ which is disjoint from $W^{s}\left(\overline{\varphi_{t}}\right)$. Let $D=F_{[0,1]}\left(S^{1}\right) \cup D_{1}$ which is also a disk in $\partial_{1} \bar{N}$. It is easy to check that $\partial D=c$ and $D \cap W^{s}\left(\overline{\varphi_{t}}\right)=\emptyset$.

Let $\Sigma_{g, k}$ be a genus $g$ orientable surface with $k$ punctures. We endow $\Sigma_{g, k} \times[0,1]$ with the vector field $\frac{\partial}{\partial t}$. Let $\bar{T}$ be a thickened template and denote the set of dividing curves by set $C=\left\{c_{1}, c_{2}, \ldots, c_{n}\right\}$ where each $c_{i}(i=1, \ldots, n)$ is a simple closed curve. Consider a partition of $C$, i.e., $C=C_{1} \sqcup C_{2} \sqcup \ldots \sqcup C_{t}$ satisfying $C_{j} \subset C(j=1, \ldots, t)$ and $C_{i} \cap C_{j}=\emptyset(i \neq j)$. Denote the number of the connected components of $C_{i}$ by $k_{i}$. One may attach $\Sigma_{g_{i}, k_{i}} \times[0,1]$ to $\bar{T}$ along $C_{i}$ similar to attaching 2-handle. By Proposition 2.12 and Theorem 4.3, we have the following theorem.

Theorem 4.4. If $U$ is a filtrating neighborhood with a basic set modeled by $T$, then there exists a partition $C_{1} \sqcup C_{2} \sqcup \ldots \sqcup C_{t}$ of $C$ such that $U$ is topologically equivalent to $\left(\phi_{t}, N\right)$. Here $\left(\phi_{t}, N\right)$ is obtained by attaching $\Sigma_{g_{i}, k_{i}} \times[0,1]$ to $\bar{T}$ along $C_{i}(i=1, \ldots, t)$ for some nonnegative integers $g_{i}(i=1, \ldots, t)$.

Theorem 4.5. Let $M=M^{\prime} \sharp m S^{1} \times S^{2}$ where $M^{\prime}$ is prime to $S^{1} \times S^{2}$. Suppose $\phi_{t}$ is an NS flow on $M$ with a basic set $\Lambda$ modeled by a template $T$. As Theorem 4.4 shows, the filtrating neighborhood $U$ of $\Lambda$ is obtained by attaching $\Sigma_{g_{i}, k_{i}} \times[0,1]$ $(i=1, \ldots, t)$ to $\bar{T}$ along dividing curves set $C$ of $\bar{T}$. Then for any $i \in\{1, \ldots, t\}$, we have $g_{i} \leq m+1$ and

(1) $k_{i} \leq 4 m-3 g_{i}+3$ for $g_{i} \leq m$; 
(2) $k_{i} \leq m+1$ for $g_{i}=m+1$.

Proof. By Theorem 1 of [27], the genus of any connected component of $\partial U$ is no more than $m+1$, hence $g_{i} \leq m+1$ for any $i \in\{1, \ldots, t\}$.

Suppose $\Sigma_{g_{i}, k_{i}} \times 0\left(\Sigma_{g_{i}, k_{i}} \times 1\right)$ is attached to $\Sigma_{0}\left(\Sigma_{1}\right)$ which is a connected component of $\partial U$. Under the assumption $k_{i} \geq 4 m-3 g_{i}+4$, we obtain the following two claims.

Claim 1. There exist at least $r\left(g_{i}\right)=2 m-g_{i}+2$ simple closed curves of $C_{i} \times 1$ such that these curves bound the disks $D_{1}, D_{2}, \ldots, D_{r\left(g_{i}\right)}$ in $\Sigma_{1}$.

Otherwise, there exist $k=2 m-2 g_{i}+3$ simple closed curves in $C_{i} \times 1$ bound some surfaces $S_{1}, S_{2}, . ., S_{s}$ in $\Sigma_{1}$ such that none of them is homeomorphic to a disk. Without loss of generality, we suppose these simple closed curves are $c_{1}, \ldots, c_{k}$. If $\partial S_{j}(j=1, . ., s)$ is connected, then the genus of $S_{j}$ is at least 1 . Denote the number of the connected components of $\partial S_{j}$ by $r_{j}$. If $\partial S_{j}$ isn't connected, the genus of $\left(\Sigma_{1}-S_{j}\right)$ is at most $m+1-r_{j}+1$. Suppose none of $\partial S_{1}, \ldots, \partial S_{\lambda}(\lambda \leq s)$ is connected and each of $\partial S_{\lambda+1}, \ldots, \partial S_{s}$ is connected. It is easy to show that the genus of $\Sigma_{g_{i}, k_{i}}$ is at most

$$
\begin{aligned}
& m+1-\sum_{j=1}^{\lambda}\left(r_{j}-1\right)-(s-\lambda) \\
& =m+1-k+\lambda \quad\left(\text { since } \sum_{j=1}^{\lambda} r_{j}+(s-\lambda)=k\right) \\
& =2 g_{i}-m-2+\lambda \quad\left(\text { since } k=2 m-2 g_{i}+3\right) .
\end{aligned}
$$

Noting that $r_{j} \geq 2$ and $\sum_{j=1}^{\lambda} r_{j}+(s-\lambda)=k=2 m-2 g_{i}+3$, we have $\lambda \leq m-g_{i}+1$ and $2 g_{i}-m-2+\lambda \leq 2 g_{i}-m-2+\left(m-g_{i}+1\right)=g_{i}-1$. Therefore, the genus of $\Sigma_{g_{i}, k_{i}}$ is at most $g_{i}-1$. It contradicts the fact that the genus of $\Sigma_{g_{i}, k_{i}}$ is $g_{i}$.

Claim 2. The disks $D_{1}, \ldots, D_{r\left(g_{i}\right)}$ together with the backward trajectory of their boundaries and $\Sigma_{0}-\Sigma_{g_{i}, k_{i}} \times 0\left(0 \leq g_{i} \leq m+1\right)$ compose at least $m+12$-spheres.

Case 1: $g_{i}=m+1$.

By the assumption " $k_{i} \geq 4 m-3 g_{i}+4$ " and Claim 1, we have $k_{i} \geq m+1$ and there exist at least $r(m+1)=m+1$ simple closed curves of $C_{i} \times 1$ which bound disks in $\Sigma_{1}$. By Theorem 1 of [27], the genus of $\Sigma_{0}$ and $\Sigma_{1}$ are no more than $m+1$, so the backward trajectory of the boundaries of $D_{1}, \ldots, D_{r\left(g_{i}\right)}$ also bounds disks in $\Sigma_{0}$. Therefore, if $g_{i}=m+1$, Claim 2 is true.

Case 2: $g_{i} \leq m$ and $C_{i} \times 0$ bound some genus 0 surfaces $F_{1}, F_{2}, \ldots, F_{\mu}$ in $\Sigma_{0}$.

Suppose $\partial F_{i}(i=1, . ., \mu)$ has $r_{i}\left(r_{i} \geq 1\right)$ connected components. Obviously $g_{i}=m+1-\sum_{i=1}^{\mu}\left(r_{i}-1\right)=m+\mu+1-\sum_{i=1}^{\mu} r_{i}$. By Claim 1, there exist at least $r\left(g_{i}\right)=2 m-g_{i}+2=2 m-(m+\mu+1)+\sum_{i=1}^{\mu} r_{i}+2=(m+1)+\sum_{i=1}^{\mu}\left(r_{i}-1\right)$ simple closed curves of $C_{i} \times 1$ bound disks $D_{1}, D_{2}, \ldots, D_{r\left(g_{i}\right)}$ in $\Sigma_{1}$. Since $\partial F_{i}$ $(i=1, . ., \mu)$ has $r_{i}\left(r_{i} \geq 1\right)$ connected components, the genus of $F_{i}$ is 0 and $r\left(g_{i}\right)=$ $(m+1)+\sum_{i=1}^{\mu}\left(r_{i}-1\right)$. Therefore, the backward trajectory of these simple closed curves (the boundaries of $\left.D_{1}, D_{2}, \ldots, D_{r\left(g_{i}\right)}\right)$ bounds at least $m+1$ disks in $\Sigma_{0}$. Therefore, in this case, Claim 2 is also true.

Case 3: $g_{i} \leq m$ and there exists $\left\{c_{1} \times 0, \ldots, c_{k} \times 0\right\} \subset C_{i} \times 0$ bounds a genus $g$ $(g \geq 1)$ surface in $\Sigma_{0}$.

Attaching a surface $\Sigma_{g, k}$ to $\Sigma_{g_{i}, k_{i}}$ along its boundary, we have a surface $\Sigma^{\prime}$. The genus of $\Sigma^{\prime}$ is $g_{i}+g+k-1$ and $\partial \Sigma^{\prime}$ has at least $4 m-3 g_{i}+4-k$ components. 
By Claim 1, there exist at least $r\left(g_{i}\right)=2 m-g_{i}+2$ simple closed curves of $C_{i} \times 1$ which bound disks in $\Sigma_{1}$, so there exist at least $2 m-g_{i}+2-k$ simple closed curves of $\partial \Sigma^{\prime} \times 1$ which bound disks in $\Sigma_{1}$.

Since the genus of $\Sigma^{\prime}$ is $g_{i}+g+k-1, \partial \Sigma^{\prime}$ has at least $4 m-3 g_{i}+4-k$ $\left(>4 m-3\left(g_{i}+g+k-1\right)+4\right)$ components and there exist at least $2 m-g_{i}+2-k$ $\left(>2 m-\left(g_{i}+g+k-1\right)+2\right)$ simple closed curves of $\partial \Sigma^{\prime} \times 1$ which bound disks in $\Sigma_{1}$. By induction on the number of the connected components of $\Sigma_{0}-\Sigma \times 0$ whose genus is no less than 1 , it can always be reduced to a situation similar to $g_{i} \leq m+1$ or a situation in which $g_{i} \leq m$ and $C_{i} \times 0$ bound some genus 0 surfaces. Here $\Sigma \times 0 \subset \Sigma_{0}$. By the previous discussions, in these two situations, Claim 2 is true. Therefore, Claim 2 is always true.

Now, let's return to the proof of the theorem. Let $S_{1}^{2}, \ldots, S_{m+1}^{2}$ be $m+12$-spheres in Claim 2, since $M=M^{\prime} \sharp m S^{1} \times S^{2}$ where $M^{\prime}$ is prime to $S^{1} \times S^{2}$, there exists $r \in \mathbb{N}$ satisfying $r \leq m+1$ such that $S=S_{1}^{2} \cup S_{2}^{2} \cup \ldots \cup S_{r}^{2}$ divides $M$ into two connected components.

If $g_{i} \leq m$ and $k_{i} \geq 4 m-3 g_{i}+4 \geq m+4$, then $S$ borders with $\bar{T}$ on both sides of $S$, the template $T$ can't be connected. It is a contradiction. This means " $k_{i} \geq 4 m-3 g_{i}+4$ " is impossible, therefore $k_{i} \leq 4 m-3 g_{i}+3$.

If $g_{i}=m+1$ and $k_{i} \geq m+2$, then $m+2>4 m-3(m+1)+4=4 m-3 g_{i}+4$. Therefore, Claim 2 is also true in this case. Since $k_{i} \geq m+2, S$ borders with $\bar{T}$ on both sides of $S$, the template $T$ can't be connected. It is a contradiction, so $k_{i} \leq m+1$ when $g_{i}=m+1$.

Corollary 4.6. There exist at most a finite number of filtrating neighborhoods (up to topological equivalence) for a given closed orientable 3-manifold $M$ and a template $T$ such that:

(1) $T$ models the invariant sets of these filtrating neighborhoods;

(2) Everyone of these filtrating neighborhoods can be realized as a filtrating neighborhood of an NS flow on $M$.

Proof. Suppose $U$ is a filtrating neighborhood with basic set modeled by $T$ and can be embedded into $M$. By Theorem 4.4,U is topologically equivalent to $\left(\phi_{t}, N\right)$ which is obtained by attaching $\Sigma_{g_{i}, k_{i}} \times[0,1]$ to $\bar{T}$ along $C_{i}(i=1, \ldots, t)$ for a partition $C_{1} \sqcup C_{2} \sqcup \ldots \sqcup C_{t}$ of $C$. Here $C$ is the dividing curves set of $\bar{T}$ and $k_{i}$ is the number of connected components of $C_{i}$. Suppose $M \cong M^{\prime} \sharp m S^{1} \times S^{2}$ where $M^{\prime}$ is prime to $S^{1} \times S^{2}$. Obviously, the partition number of $\left\{c_{1}, \ldots, c_{n}\right\}$ is finite. By Theorem 4.5, $g_{i} \leq m+1$ for any $i \in\{1,2, \ldots, t\}$. We can obtain that $\sum_{i=1}^{t} k_{i}=n$. Therefore, the number of all the possibilities of $\left(\Sigma_{g_{1}, k_{1}}, \ldots, \Sigma_{g_{t}, k_{t}}\right)$ is finite. This means the number of all the possibilities of the topological equivalence classes of $U$ is finite.

Example 4.7. Suppose $T=\mathcal{L}(1,1)$ is a template as Figure $12-1$ shows. It is a Lorenz like template, see [13] or [26]. The thickened template $\bar{T}$ of $T$ is shown in Figure 12-2 and Figure 12-3. $X$ and $Y$ are the exit set and the entrance set of $\partial \bar{T}$ respectively. $\{a, b, c\}$ is the dividing curves set of $\bar{T}$. Figure $12-4$ shows $X \cup\{a, b, c\}$.

Let $M$ be a closed orientable irreducible 3 -manifold and $\left(\phi_{t}, N\right)$ be a filtrating neighborhood with invariant set modeled by $T$ which can be realized as a filtrating neighborhood of an NS flow on $M$. Noting that $a$ and $b$ are symmetric in $\bar{T}$ and $M$ is prime to $S^{1} \times S^{2}$, by a proof similar to that of Corollary 4.6, we know that $\left(\phi_{t}, N\right)$ may be obtained by one of the following procedures: 


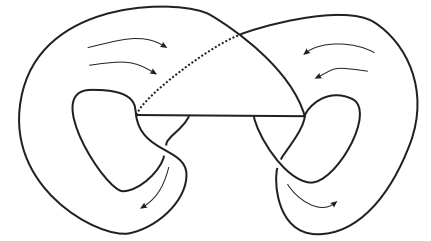

$12-1 \mathrm{~L}(1,1)$

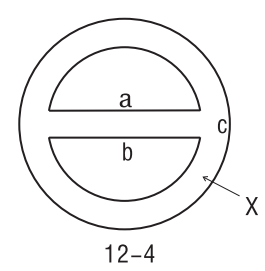

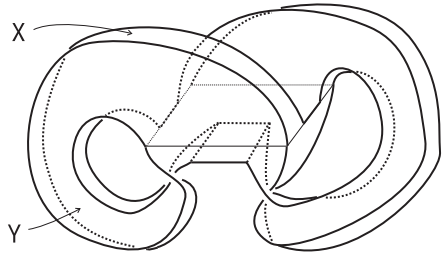

12-2 thickened tempate of $L(1,1)$

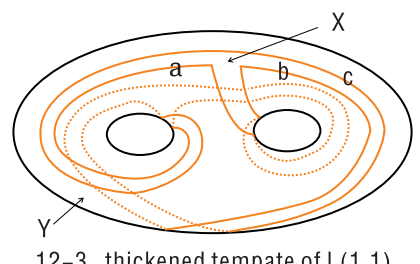

FiguRe 12.

(1) attaching $\Sigma_{0,2} \times[0,1]$ to $\bar{T}$ along $\{a, b\}$ and $\Sigma_{0,1} \times[0,1]$ to $\bar{T}$ along $\{c\}$;

(2) attaching $\Sigma_{0,2} \times[0,1]$ to $\bar{T}$ along $\{a, c\}$ and $\Sigma_{0,1} \times[0,1]$ to $\bar{T}$ along $\{b\}$;

(3) attaching $\Sigma_{1,1} \times[0,1]$ to $\bar{T}$ along $\{a\}$ and two $\Sigma_{0,1} \times[0,1]$ to $\bar{T}$ along $\{b\}$ and $\{c\}$;

(4) attaching $\Sigma_{1,1} \times[0,1]$ to $\bar{T}$ along $\{c\}$ and two $\Sigma_{0,1} \times[0,1]$ to $\bar{T}$ along $\{a\}$ and $\{b\}$.

In case (1) and (2) above, by the proofs of Theorem 2 and Theorem 4 in [26], it is easy to show that $N \cong L(3,1) \sharp T^{2} \times[0,1] . \quad M$ is irreducible and $N$ can be embedded into $M$, therefore $M \cong L(3,1)$ and the interior of $N \cong(L(3,1)-K)$. Here $K$ is a Hopf link in a three ball in $L(3,1)$.

In case (3) and (4) above, also by the proofs of Theorem 2 and Theorem 4 in [26], we have that $N \cong S^{1} \times D^{2}-V$. Here $V$ is a small open neighborhood of a standard $(3,1)$ torus knot in the interior of $S^{1} \times D^{2}$. Since $N$ can be realized as a filtrating neighborhood of an NS flow on $S^{1} \times D^{2}$ and any closed orientable 3-manifold can admit an NS flow ([27]), $M$ may be any closed orientable irreducible 3-manifold. In particular, if $M \cong S^{3}$ and $N$ can be realized as a filtrating neighborhood of an NS flow on $S^{3}$, by the proofs of Theorem 2 and Theorem 4 in [26], $S^{3}-N$ may be a small open neighborhood of a two components link $L$ which is composed of a trivial knot and a $(p, 3)$ torus knot in the boundary of a solid torus neighborhood of the trivial knot. Here $p$ is any integer such that $p$ and 3 are coprime.

Remark 4.8. Example 4.7 tells us that, given a closed orientable 3-manifold $M$ and a template $T$, although there exist at most a finite number of filtrating neighborhoods with invariant set modeled by $T$ such that each of them can be realized as a filtrating neighborhood of an NS flow on $M$, there may be infinitely many different ways to embed some filtrating neighborhood of $T$ as a filtrating neighborhood of an NS flow on $M$.

\section{Embedding Templates in NS Flows on 3-Manifolds}

To prove the main theorem (Theorem 5.5) of this section, we first recall a theorem due to J. Morgan [16]: 
Theorem 5.1. Suppose that $M$ is an orientable 3-manifold with $X\left(M, \partial_{-} M\right)=0$ where $\partial_{-} M$ is composed of some connected components of $\partial M$ and $X\left(M, \partial_{-} M\right)$ is the Euler number of $\left(M, \partial_{-} M\right)$. For some nonnegative integer $k,\left(M \sharp k S^{1} \times\right.$ $\left.S^{2}, \partial_{-} M\right)$ has an NMS flow which is transverse outside to $\partial_{-} M$ and transverse inside to $\partial M-\partial_{-} M$.

Lemma 5.2. Let $W$ be a compact 3-manifold with boundary $\Sigma \cup S_{1} \cup \ldots \cup S_{g}$ where $\Sigma$ is a genus $(g+1)$ closed orientable surface and each $S_{i}(i=1, \ldots, g)$ is homeomorphic to a 2-shpere. Then for some nonnegative integer $k$, there exists an NMS flow $\phi_{t}$ on $W \sharp k S^{1} \times S^{2}$ such that $\phi_{t}$ is transverse outside to $\partial\left(W \sharp k S^{1} \times S^{2}\right)=\Sigma \cup S_{1} \cup \ldots \cup S_{g}$.

Proof. Attaching $W$ to a copy of $W$ along $\partial W$, we obtain a closed 3-manifold $M$. Obviously $X(M)=0=2 X(W, \partial W)-X(\partial W)$. On the other hand,

$$
X(\partial M)=\sum_{i=1}^{g} X\left(s_{i}\right)+X(\Sigma)=2 g+(2-2(g+1))=0 .
$$

Then we have $X(W, \partial W)=0$, hence $(W, \partial W)$ satisfies the condition of Theorem 5.1. By Theorem 5.1, for some nonnegative integer $k, W \sharp k S^{1} \times S^{2}$ admits an NMS flow $\phi_{t}$ which is transverse outside to $\partial W$.

Theorem 5.3. (Meleshuk [17]) Any template $T$ can model a basic set $\Lambda$ of a Smale flow $\varphi_{t}$ on $S^{3}$.

Lemma 5.4. There exists an $N S$ flow $f_{t}$ on $S^{2} \times I$ such that:

(1) $f_{t}$ is transverse inward to $S^{2} \times 0$ and outward to $S^{2} \times 1$;

(2) there is no flowline which starts in $S^{2} \times 0$ and terminates in $S^{2} \times 1$.

Proof. In the proof of Lemma 3.4 of [27], choosing $n=1$, we get a Smale flow $\varphi_{t}$ on $S^{3}$ with two singularities: one is a sink, the other is a source. Cutting two standard small neighborhoods of the sink and the source respectively, we obtain an NS flow $f_{t}$ on $S^{2} \times I$ which satisfies (1) of Lemma 5.4. By the argument in the proof of Lemma 3.4 of [27], it is easy to prove that there is no flowline of $f_{t}$ which starts in $S^{2} \times 0$ and terminates in $S^{2} \times 1$.

Theorem 5.5. Let $T$ be a template. Then there exists a positive integer $n$ such that $n S^{1} \times S^{2}$ admits an NS flow $\psi_{t}$ with a basic set $\Lambda$ modeled by $T$.

Proof. Let $\left(\phi_{t}, N\right)$ be a filtrating neighborhood of $\Lambda$ of $\varphi_{t}$ on $S^{3}$ where $\varphi_{t}$ is the Smale flow in Theorem 5.3. Suppose $\partial N=\Sigma_{1}^{+} \cup \ldots \cup \Sigma_{t}^{+} \cup \Sigma_{1}^{-} \cup \ldots \cup \Sigma_{s}^{-}$where $\Sigma_{1}^{+} \cup \ldots \cup \Sigma_{t}^{+}$and $\Sigma_{1}^{-} \cup \ldots \cup \Sigma_{s}^{-}$are the entrance set and exit set of $\varphi_{t}$ on $N$ respectively.

Suppose

$$
\begin{aligned}
& g_{i}^{+}>1, i=1, \ldots, t_{1} \\
& g_{i}^{+}=0, i=t_{1}+1, \ldots, t_{1}+t_{2} \\
& g_{i}^{+}=1, i=t_{1}+t_{2}+1, \ldots, t
\end{aligned}
$$

and

$$
\begin{aligned}
& g_{j}^{-}>1, j=1, \ldots, s_{1} \\
& g_{j}^{-}=0, j=s_{1}+1, \ldots, s_{1}+s_{2} \\
& g_{j}^{-}=1, j=s_{1}+s_{2}+1, \ldots, s .
\end{aligned}
$$


Here each of $t_{1}, t_{2}, t-t_{1}-t_{2}, s_{1}, s_{2}$ and $s-s_{1}-s_{2}$ is a nonnegative integer and $g_{i}^{+}\left(g_{j}^{-}\right)$is the genus of $\Sigma_{i}^{+}\left(\Sigma_{j}^{-}\right)$.

Since any embedded surface in $S^{3}$ is separable, $\overline{S^{3}-N}=M_{1}^{+} \sqcup \ldots \sqcup M_{t}^{+} \sqcup M_{1}^{-} \sqcup$ $\ldots \sqcup M_{s}^{-}$where $\partial M_{i}^{+}=\Sigma_{i}^{+}$and $\partial M_{j}^{-}=\Sigma_{j}^{-}$. Cutting $g_{i}^{+}-1$ open 3 -balls in $M_{i}^{+}$ $\left(i=1, \ldots, t_{1}\right)$, we get a compact 3 -manifold $W_{i}^{+}$such that $\partial W_{i}^{+}$is composed of $\Sigma_{i}^{+}$ and $g_{i}^{+}-12$-spheres. Similarly, cutting $g_{j}^{-}-1$ open 3 -balls in $M_{j}^{-}\left(j=1, \ldots, s_{1}\right)$, we get a compact 3 -manifold $W_{j}^{-}$such that $\partial W_{j}^{-}$is composed of $\Sigma_{j}^{-}$and $g_{j}^{-}-1$ 2 -spheres. By Lemma 5.2, there exist NMS flows $\phi_{i}^{+}(t)\left(i=1, \ldots, t_{1}\right)$ and $\phi_{j}^{-}(t)$ $\left(j=1, \ldots, s_{1}\right)$ on $W_{i}^{+} \sharp m_{i} S^{1} \times S^{2}$ and $W_{j}^{-} \sharp n_{j} S^{1} \times S^{2}$ respectively such that $\phi_{i}^{+}(t)$ is transverse outside to $\partial W_{i}^{+}$and $\phi_{j}^{-}(t)$ is transverse inside to $\partial W_{j}^{-}$.

When $t_{1}+t_{2}+1 \leq i \leq t$ and $s_{1}+s_{2}+1 \leq j \leq s, \partial M_{i}^{+} \cong \partial M_{j}^{-} \cong T^{2} \cdot M_{i}^{+}\left(M_{j}^{-}\right)$ can be embedded into $S^{3}$, so $M_{i}^{+}\left(M_{j}^{-}\right)$is a knot complement. By Proposition 6.1 of J. Franks [7], there exists an NS flow $\phi_{i}^{+}(t)\left(\phi_{j}^{-}(t)\right)$ on $M_{i}^{+}\left(M_{j}^{-}\right)$which is transverse inside (outside) to $\partial W_{i}^{+}\left(\partial W_{j}^{-}\right)$.

By attaching $\left(W_{i}^{+} \sharp m_{i} S^{1} \times S^{2}, \phi_{i}^{+}(t)\right)\left(i=1, \ldots, t_{1}\right),\left(W_{j}^{-} \sharp n_{j} S^{1} \times S^{2}, \phi_{j}^{-}(t)\right)(j=$ $\left.1, \ldots, s_{1}\right),\left(M_{i}^{+}, \phi_{i}^{+}(t)\right)\left(i=t_{1}+t_{2}+1, \ldots, t\right)$ and $\left(M_{j}^{-}, \phi_{j}^{-}(t)\right)\left(j=s_{1}+s_{2}+1, \ldots, s\right)$ to $\left(N, \phi_{t}\right)$ standardly, we get an NS flow $\psi_{t}^{1}$ on $V$ where $V$ is homeomorphic to $\left(\sum_{i=1}^{t_{1}} m_{i}+\sum_{j=1}^{s_{1}} n_{j}\right) S^{1} \times S^{2}$ with $\left(\sum_{i=1}^{t_{1}} g_{i}^{+}-t_{1}\right)+\left(\sum_{j=1}^{s_{1}} g_{j}^{-}-s_{1}\right)+t_{2}+s_{2}$ punctures. By Poincáre-Hopf theorem, it is easy to show that $\sum_{i=1}^{t_{1}} g_{i}^{+}-t_{1}+s_{2}=$ $\sum_{j=1}^{s_{1}} g_{j}^{-}-s_{1}+t_{2}$ (denote this number by $r$ ). So $\left(V, \psi_{t}^{1}\right)$ is transverse outside to 2-spheres $S_{1}^{+}, \ldots, S_{r}^{+}$and inside to 2-spheres $S_{1}^{-}, \ldots, S_{r}^{-}$.

If either $S_{i}^{+}$belongs to some $W_{i}^{+} \sharp m_{i} S^{1} \times S^{2}$ or $S_{i}^{-}$belongs to some $W_{j}^{-} \sharp n_{j} S^{1} \times$ $S^{2}$, we attach $S_{i}^{+}$to $S_{i}^{-}$. Otherwise, we attach $\left(S^{2} \times I, f_{t}\right)$ in Lemma 5.4 to $\left(V, \psi_{t}^{1}\right)$ such that $S^{2} \times 0$ is attached to $S_{i}^{+}$and $S^{2} \times 1$ is attached to $S_{i}^{-}$.

Thus we obtain a flow $\varphi_{t}$ on $n S^{1} \times S^{2}$ where $n=\sum_{i=1}^{t_{1}} m_{i}+\sum_{j=1}^{s_{1}} n_{j}+r$. Noting that (2) in Lemma 5.4 ensures that there are no more chain recurrent points except the chain recurrent sets which we have controlled, it is easy to check that $\varphi_{t}$ is an NS flow.

By a comparison of Theorem 5.5 and the proof of Lemma 3.6 in [27], we have the following corollary.

Corollary 5.6. Let $T$ be a template. There exists a positive integer $n$ such that, for any closed orientable 3-manifold $M^{\prime}$, there exists an NS flow $\psi_{t}$ on $M=M^{\prime} \sharp n S^{1} \times$ $S^{2}$ with a basic set $\Lambda$ modeled by $T$.

Furthermore, the following question is natural.

Question 5.7. Let $T$ be a template. Does there exist a positive integer $n$ such that any closed orientable 3-manifold $M$ admits an NS flow with a basic set modeled by $T$ if and only if $M=M^{\prime} \sharp n S^{1} \times S^{2}$ ? Here $M^{\prime}$ is any closed orientable 3-manifold.

\section{ACKNOWLEDGMENTS}

The author would like to thank Hao Yin for his many helpful suggestions and comments. The author also would like to thank the referee for carefully reading this paper and providing many helpful suggestions and comments. 


\section{REFERENCES}

1. D. Asimov, Round handles and non-singular Morse-Smale flows, Ann. of Math. (2), 102 (1975), no. 1, 41-54.

2. F. Béguin, C. Bonatti, Flots de Smale en dimension 3: prsentations finies de voisinages invariants d'ensembles selles, (French) [Smale flows in dimension 3: finite presentations of invariant neighborhoods of saddle sets], Topology, 41 (2002), no. 1, 119-162.

3. R. Bowen, One-dimensional hyperbolic sets for flows, J. Differential Equations, 12 (1972), 173-179.

4. R. Bowen, P. Walters, Expansive one-parameter flows, J. Differential Equations, 12 (1972), 180-193.

5. J. Birman, R. F. Williams, Knotted periodic orbits in dynamical systems. I. Lorenz's equations, Topology, 22 (1983), no. 1, 47-82.

6. J. Birman, R. F. Williams, Knotted periodic orbits in dynamical system. II. Knot holders for fibered knots, in "Low-dimensional topology" (San Francisco, Calif., 1981), 1-60, Contemp. Math., 20, Amer. Math. Soc., Providence, RI, 1983.

7. J. Franks, Knots, links and symbolic dynamics, Ann. of Math. (2), 113 (1981), no. 3, 529-552.

8. J. Franks, "Homology and Dynamical Systems," CBMS49.American Mathematical Society. Providence, Rhode Island,1982.

9. J. Franks, Symbolic dynamics in flows on three-manifolds, Trans. Amer. Math. Soc, 279 (1983), no. 1, 231-236.

10. J. Franks, Flow equivalence of subshifts of finite type, Ergodic Theory Dynam. Systems, 4 (1984), no. 1, 53-66.

11. J. Franks, Nonsingular Smale flows on $S^{3}$, Topology, 24 (1985), no. 3, 265-282.

12. G. Frank, Templates and train tracks, Trans. Amer. Math. Soc, 308 (1988), no. 2, 765-784.

13. R. W. Ghrist, P. J. Holmes and M. C. Sullivan, "Knots and Links in Three-dimensional Flows," Lecture Notes in Mathematics, 1654. Springer-Verlag, Berlin, 1997.

14. L. Kauffman, M. Saito and M. Sullivan, Quantum invariants of templates, J. Knot Theory Ramifications, 12 (2003), no. 5, 653-681.

15. D. Lind, B. Marcus, "An Introduction to Symbolic Dynamics and Coding," Cambridge University Press, Cambridge, 1995.

16. J. Morgan, Nonsingular Morse-Smale flows on 3-dimensional manifolds, Topology, 18 (1978), 41-54.

17. V. Meleshuk, "Embedding Templates in Flows," Ph.D thesis, Northwestern University, 2002.

18. B. Parry, D. Sullivan, A topological invariant of flows on 1-dimensional spaces, Topology, 14 (1975), no. 4, 297-299.

19. C. Pugh, M. Shub, Suspending subshifts, in "Contributions to analysis and geometry" (eds. Md. Baltimore), Johns Hopkins Univ. Press, (1981), 265-275.

20. K. de Rezende, Smale flows on the three-sphere, Trans. Amer. Math. Soc, 303 (1987), no. 1, 283-310.

21. C. Robinson, "Dynamical Systems. Stability, Symbolic Dynamics, and Chaos," $2^{\text {nd }}$ edition, Studies in Advanced Mathematics. CRC Press, Boca Raton, FL, 1999.

22. S. Smale, Differentiable dynamical systems, Bull. Amer. Math. Soc, 73 (1967), 747-817.

23. M. C. Sullivan, Visually building Smale flows on $S^{3}$, Topology Appl, 106 (2000), no. 1, 1-19.

24. M. Wada, Closed orbits of nonsingular Morse-Smale flows on $S^{3}$, J. Math. Soc. Japan, 41 (1989), no. 3, 405-413.

25. R. Williams, Classification of subshifts of finite type, Ann. of Math. (2), 98 (1973), 120-153; errata, ibid. (2) 99 (1974), 380-381.

26. B. Yu, Lorenz like Smale flows on three-manifolds, Topology Appl, 156 (2009), no. 15, 24622469.

27. B. Yu, Regular level sets of Lyapunov graphs of nonsingular Smale flows on 3-manifolds, Discrete Contin. Dyn. Syst, 29 (2011), no. 3, 1277-1290. doi:10.3934/dcds.2011.29.1277

Department of Mathematics, Tongu University, Shanghai, China 20092 